\title{
ENFERMEDAD DE POTT. REPORTE DE CASO CLÍNICO Y REVISIÓN BIBLIOGRÁFICA
}

\section{POTT'S DISEASE. CLINICAL CASE REPORT AND BIBLIOGRAPHIC REVIEW}

\section{Referencia de reporte de caso}

Corleto Molina, G., López España, E., \& González Morales, C. (2021). Enfermedad de Pott. Reporte de caso clínico y revisión y bibliográfica. Revista Científica del Sistema de Estudios de Postgrado de la Universidad de San Carlos De Guatemala, 4(1), $117-124$.

DOI: https://doi.org/10.36958/sep.v4i1.73

\author{
Dra. Gabriela Corleto Molina \\ gaby813corleto@gmail.com \\ D 0000-0002-2766-3598 \\ Médica y Cirujana del Centro Universitario del Norte \\ Universidad de San Carlos de Guatemala \\ Dra. Erza López España \\ Médica y Cirujana del Centro Universitario del Norte \\ Universidad de San Carlos de Guatemala
}

Mtra. Celeste González Morales

Médica y Cirujana de la Facultad de Ciencias Médicas

Universidad de San Carlos de Guatemala
Fecha de recepción: 23/05/2021

\begin{abstract}
RESUMEN
La tuberculosis es un problema sanitario importante en países en vías del desarrollo y la población pediátrica es un grupo susceptible. La enfermedad de Pott provoca destrucción ósea, deformidad espinal y complicaciones neurales. Se presenta el caso de paciente femenina de 4 años y 5 meses con enfermedad de Pott, con destrucción de cuerpos vertebrales, deformidad ósea y paraparesias, en el departamento de Pediatría del Hospital Regional de Cobán, el diagnóstico se realizó clinicoradiológico acompañado de prueba de tuberculina positiva y GeneExpert positivo. Es necesario un diagnóstico temprano y el establecimiento de un tratamiento para un buen resultado.
\end{abstract}

\section{PALABRAS CLAVE}

Tuberculosis, tuberculosis de la columna vertebral, enfermedad de Pott
Fecha de aceptación: 04/11/2021

\section{ABSTRACT}

Tuberculosis is a major health problem in developing countries and the pediatric population is a susceptible group. Pott's disease causes bone destruction, spinal deformity, and neural complications. The case of a female patient aged 4 years and 5 months with Pott's disease, with destruction of vertebral bodies, bone deformity and paraparesis, is presented in the Department of Pediatrics of the Hospital Regional de Cobán, the diagnosis was made clinicoradiologically accompanied by a tuberculin test positive and positive GeneExpert. An early diagnosis and the establishment of a treatment is necessary for a good result.

\section{KEYWORDS}

Tuberculosis, tuberculosis spinal, Pott's disease 


\section{INTRODUCCIÓN}

La tuberculosis extrapulmonar puede ser definida como aquella infección producida por Mycobacterium tuberculosis que afecta a tejidos y órganos fuera del parénquima pulmonar, representando el $20-25 \%$ de los casos de enfermedad tuberculosa, siendo la localización de afectación más frecuente, la ganglionar seguida por la urogenital y la osteoarticular. La afectación ósea y articular por tuberculosis supone el $35 \%$ de las afectaciones extrapulmonares, siendo la columna vertebral la más afectada; principalmente la columna dorsal lumbar y la dorsal baja así mismo se puede asociar a absceso paravertebral bilateral. (Barrera y Morales, 2017; Fanlo y Tiberio, 2007).

La tuberculosis vertebral o enfermedad de Pott afecta principalmente a la parte antero- inferior del cuerpo vertebral produciendo destrucción ósea y necrosis caseosa. La destrucción vertebral puede llegar a producir colapso del cuerpo vertebral y la herniación discal con deformidad en la columna en forma de cifosis o giba y compresión medular llegando a producir en ocasiones paraplejía. (Ramírez, Menéndez y Noguerado, 2015).

La Organización Mundial de la Salud (OMS) en el año 2017, reporto un millón de casos de tuberculosis en menores de 14 años, donde del $10 \%$ a $20 \%$ presentaron tuberculosis extrapulmonar, de estos, un $10 \%$ a $35 \%$ presentaron enfermedad de Pott. Debido a que uno de los factores de riesgo es el contacto con personas afectadas con tuberculosis, los niños menores de 5 años se encuentran en riesgo y son los más afectados haciendo su presentación muy variable, manifestándose clínicamente con dolor en la zona afectada, asociado a cifosis y abscesos fríos. (Cortez et al, 2018; González et al, 2014; Macias, 2017) ¿El mal de Pott puede presentarse en la población infantil de Guatemala? La importan- cia de presentar este caso clínico es que, al ser un problema de salud poco frecuente, nos sentimos motivados a resaltar algunos elementos en el diagnóstico del paciente pediátrico con enfermedad de Pott, presentado en el Hospital Regional de Cobán.

\section{Reporte de caso}

Paciente femenina de 4 años y 5 meses, originaria del municipio de Santa Cruz Verapaz, del departamento de Alta Verapaz. Sin antecedente de vacuna Bacillus-Calmatte-Guérin (BCG) al nacer y con tíos paternos con tuberculosis pulmonar activa, quienes no viven con la paciente, pero la visitan frecuentemente, se desconoce si cumplieron con tratamiento. Paciente fue llevada por madre de familia al presentar más de 4 días un cuadro generalizado de fiebre intermitente, acompañada de tos no productiva. Refiriendo más de 1 mes de pérdida de peso y el desarrollo de una giba dorsal, se le agrega debilidad de las extremidades inferiores con predominio distal. Presentando una marcada dificultad para la deambulación al ingreso al hospital.

Al examen físico se observa, palidez cutánea y piel áspera con descamación, panículo adiposo disminuido, sin adenopatías cervicales, aparato respiratorio: murmullo vesicular en ambos campos pulmonares, con frecuencia respiratoria de 19 respiraciones por minuto, sin ruidos pulmonares patológicos, aparato cardiovascular: ruidos cardiacos con buen tono e intensidad, frecuencia cardíaca de 90 latidos por minuto, sistema osteomioarticular se observa la presencia de cifosis y una giba dorsal aproximadamente a nivel de T8 y T9 (figura 1), no refiere dolor a la palpación y con afectación marcada para la deambulación, paraparesia de miembros inferiores y disminución de reflejos osteotendinosos patelares. Control de esfínteres preservado. 
Valoración nutricional: peso $9.14 \mathrm{~kg}$ y talla $84.1 \mathrm{~cm}$, indicando desnutrición proteica calórica aguda moderada.

Figura 1. Giba dorsal a nivel de T8 y T9

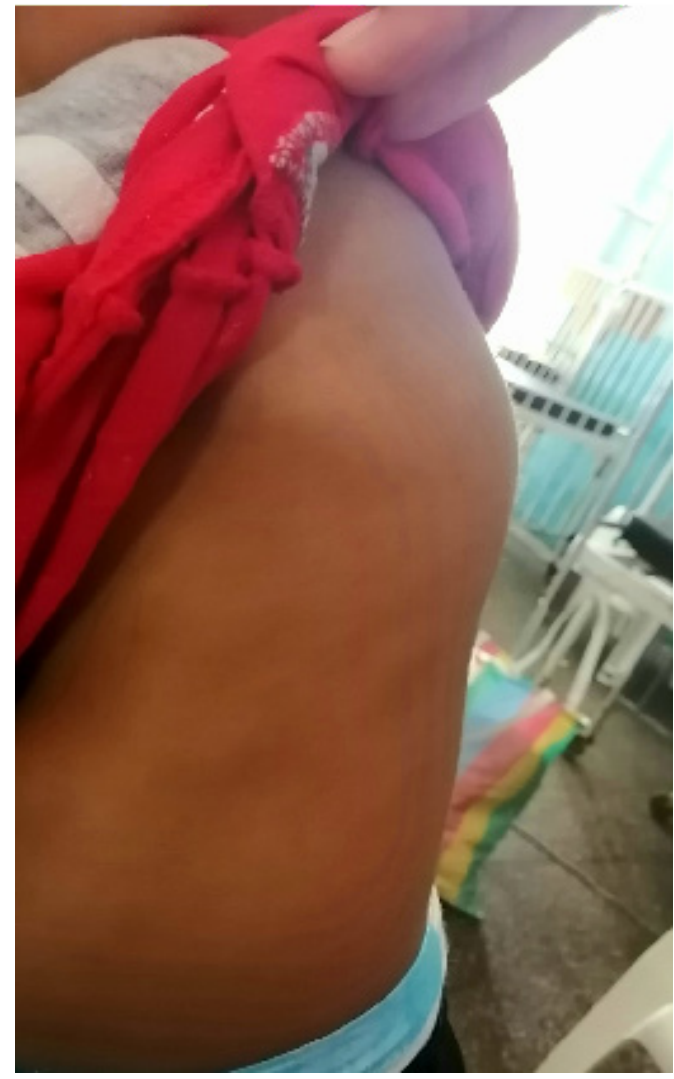

Los exámenes complementarios: hematología: leucocitosis 12.71 , NE $34.80 \%$, LIN $56.90 \%$, el test de tuberculina positiva con $>5 \mathrm{~mm}$ de induración, GeneExpert: micobacterium tuberculosis detectado, serología para VIH negativa.

En la radiografía de tórax se observan opacidades pulmonares en campo pulmonar derecho. (figura 2) En la resonancia magnética de columna dorsal: se aprecia la presencia de una imagen en reloj de arena, que destruye el cuerpo vertebral de T9, la imagen ejerce efecto de masa sobre estructuras a nivel del canal medular, provocando una disminución del diámetro anteroposterior de $67.22 \%$, su parte anterior ocupa al mediastino posterior, esta imagen es compatible con espondilodiscitis infecciosa, enfermedad de Pott. (figura $3)$.

Estos hallazgos son compatibles con enfermedad de Pott. Por lo que el paciente es referido al departamento de Neurocirugía de un hospital de tercer nivel para la descompresión quirúrgica, ya que en el Hospital Regional de Cobán no se cuenta con esta subespecialidad. 


\section{CC-S:P}

Figura 2. Radiografía de tórax lateral y anteroposterior que muestra infiltrados pulmonares en campo pulmonar derecho

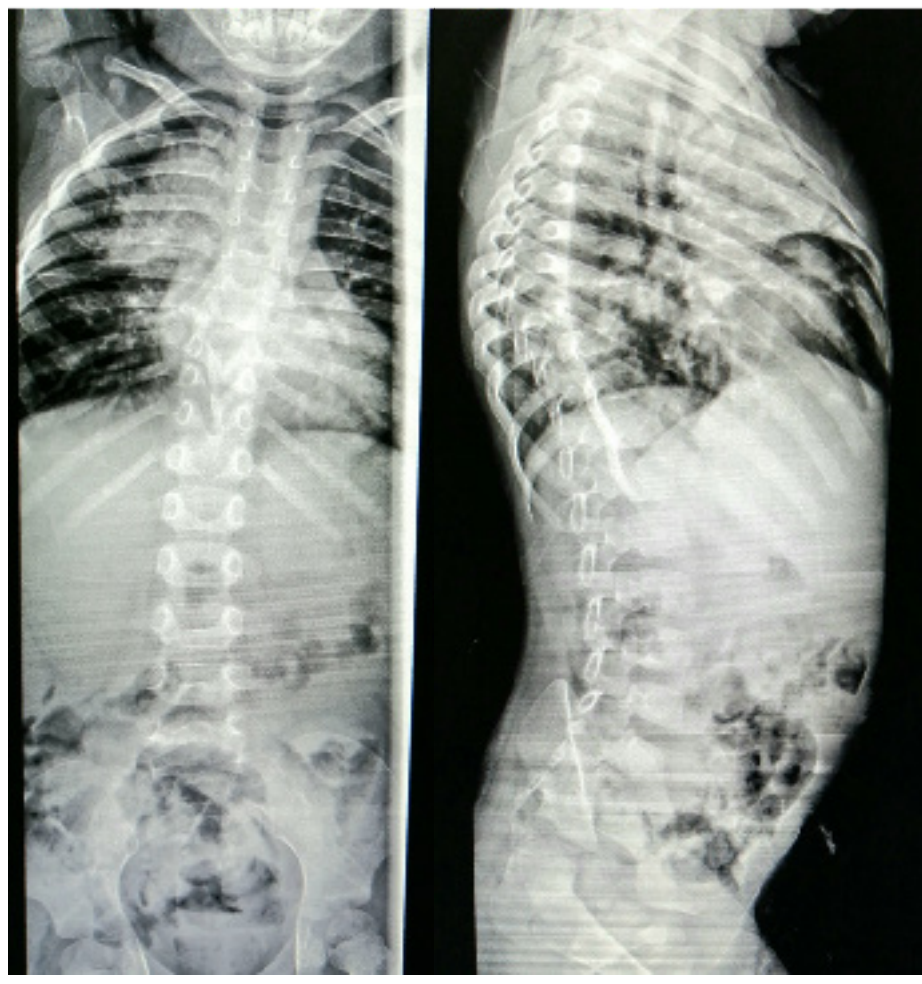

Figura 3. Resonancia magnética de columna dorsal, evidenciando imagen en reloj de arena correspondiente a espondilodiscitis infecciosa, enfermedad de Pott
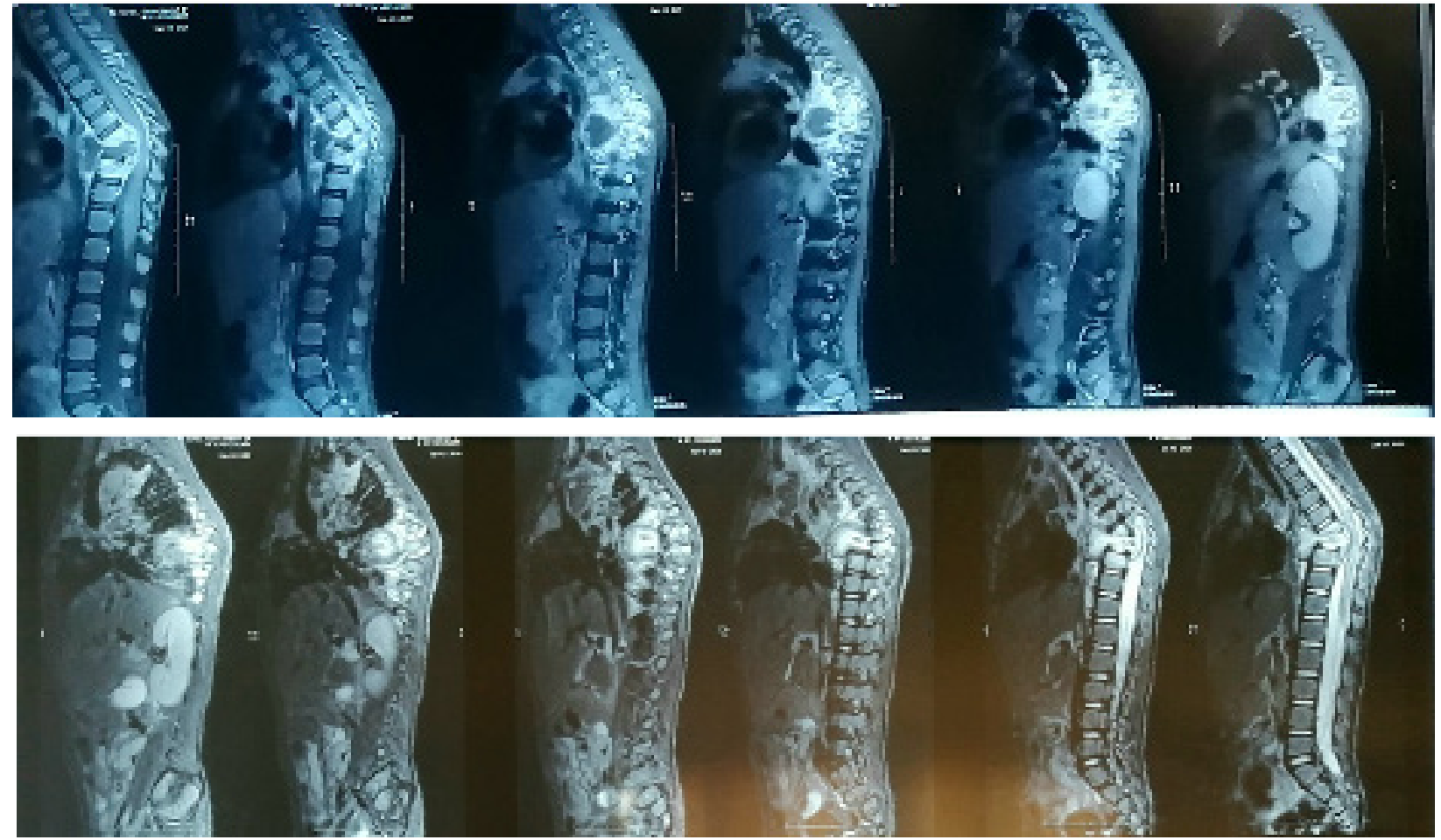


\section{DISCUSIÓN}

La enfermedad de Pott en niños es una de las principales manifestaciones de tuberculosis no pulmonar en la infancia, la mayoría de los casos se presentan en lactantes y niños menores de 5 años. En Guatemala para el año 2018 se realizó una estimación de carga de tuberculosis de 24 casos por cada 100,000 habitantes, aproximadamente 4,500 casos al año. Para el año 2019 en Guatemala se presentaron 3,568 casos con una tasa de 22 casos por cada 100,000 habitantes. (MSPAS, 2020).

La tuberculosis infantil en la mayoría de los casos se presenta cuando los menores han estado en contacto con pacientes con tuberculosis como en este caso, por lo que es importante la investigación epidemiológica y seguimiento para evitar el contagio a menores. Se sabe que la micobacteria se deposita a través de las arteriolas en el cuerpo vertebral en la parte anteroinferior produciendo destrucción ósea y necrosis caseosa, luego la infección se disemina a través del ligamento anterior afectando al cuerpo vertebral adyacente. La destrucción local puede producir el colapso del cuerpo vertebral y la herniación discal con deformidad en la columna en forma de cifosis o giba y compresión medular llegando a producir afectación en la función motora de los miembros inferiores con debilidad que puede progresar a paraplejia.

La enfermedad de Pott es el resultado de una diseminación hematógena de un sitio primario de infección, generalmente pulmonar. Estas lesiones se pueden encontrar dentro de los 3 meses a 3 años de la infancia primaria, la enfermedad pulmonar activa concomitante está presente en $<50 \%$ de los casos. La presentación clínica depende de la edad en la que se adquiere, sin embargo, es de difícil diagnóstico ya que los síntomas son de manifestación lentas e insidiosa y solo presente del 20 al 30\% de los casos. Por lo que el resto de los pacientes se pueden diagnosticar cuando están presentes las complicaciones de la enfermedad que incluyen deformidades de la columna o alteraciones en la marcha. (Cortez el al, 2018).

La evidencia científica es escasa en pediatría, los pacientes pediátricos en riesgo son aquellos que tienen dificultad de acceso a servicios de salud, desnutrición y el hacinamiento. El diagnostico en la población infantil es complejo y en nuestro contexto, el diagnostico tardío de la enfermedad se asocia a las condiciones de pobreza de los pacientes, por lo que al realizarse el diagnostico normalmente ya se presentan cambios crónicos como el caso de la paciente con presencia de la giba dorsal, tal como se observó en este caso. (Benzagmout et al, 2011; Carrol, Clark y Cant, 2001).

En la radiografía de tórax puede observarse normales o leves cambios de osteopenia, se estima que debe de haber una pérdida ósea de más del $50 \%$ para que los cambios sean evidentes en las imágenes de rayos $X$. La tomografía computarizada es útil para demostrar la esclerosis ósea y destrucción que son difíciles de observar en la radiografía convencional. (Méndez y Baquero, 2016) Sin embargo, la resonancia magnética es el examen radiológico de elección por ser más sensible y especifico ya que puede exponer masas en tejidos blandos, los discos involucrados y la compresión de la medula espinal, este puede detectar tuberculosis espinal 4-6 meses antes que cualquier otro método convencional y esta es la modalidad de elección para determinar el grado de afectación de los tejidos blandos y evaluar la respuesta a tratamientos, también es capaz de dar precisión para la planificación quirúrgica, donde en el $24 \%$ de los casos es necesaria una cirugía. Sin embargo, se recomienda individualizar cada 
caso en particular para ver el tratamiento adecuado. (Barrera y Morales, 2017).

La prueba de GeneExpert es una técnica de PCR en tiempo real, y es el método de elección diagnostica de la TB en pacientes con $\mathrm{VIH}$, y en niños con cualquiera de las presentaciones de TB infantil. Y como en el caso presentado la prueba de GeneExpert fue el método diagnostico ya que la baciloscopia dio negativa, porque en niños es difícil expectorar y muchas veces las muestras obtenidas por jugo gástrico no se obtienen en cantidades adecuadas para el diagnóstico. Es importante mencionar que en este caso el paciente presenta desnutrición proteico calórica y se sabe que la relación entre desnutrición y TB es interactiva, ya que la desnutrición expone a una invasión fácil de enfermedades infectocontagiosas y la infección por TB conduce o agrava la desnutrición. (Mellado et al, 2018; Agrons, Markowitz y Kramer, 1993)

Tabla 1. Datos relevantes para el diagnóstico

\begin{tabular}{ll}
\hline & DATOS RELEVANTES PARA EL DIAGNÓSTICO \\
\hline Factores de & Inmunocompromiso \\
riesgo & VIH \\
& Contacto reciente con pacientes \\
& Tener tuberculosis \\
\hline Signos y síntomasFiebre \\
comunes & Pérdida de peso \\
& Anormalidad neurológica: paraplejía, pérdida de sensibilidad, \\
& disfunción intestinal \\
& Transpiración nocturna \\
& Giba dorsal \\
& Dolor de espalda \\
\hline Pruebas de & Tinción de Ziehl-Neelsen sensibilidad de 59.8\% con una muestra, \\
laboratorio & 65.8\% con dos muestras y 70.5\% con tres muestras y especificidad \\
& de 98\%. \\
& Prueba de tuberculina tiene baja especificidad y sensibilidad. \\
& GeneExpert tiene una sensibilidad mayor de 82\%. \\
\hline Características & Hallazgos neuroradiológicos: \\
de imagen & Imágenes de radiografía simple que muestran apariencia de nido \\
& de pájaro, fenómeno aneurismático o resonancia magnética que \\
& muestra lesiones que se originan en la placa vertebral terminal, \\
& involucran la esquina anterior del cuerpo vertebral, muestran \\
& evidencia de diseminación subligamentosa, incluyen múltiples \\
& cuerpos vertebrales, pero discos conservados, con formación \\
& extensa de abscesos paraespinales, calcificación del absceso, \\
destrucción vertebral o colapso del cuerpo vertebral.
\end{tabular}

Fuente: Chang, Yu, Chih et al. (2016) Early diagnosis of spinal tuberbulosis. Journal of the Formosan Medical Association. 115(10):825-836. 


\section{CONCLUSIONES}

En un país con "alta carga de tuberculosis" como describe la OPS/OMS a Guatemala, es necesario sospechar enfermedad de Pott en todo niño con deformidad cifótica o dificultad de la marcha. Las pruebas inmunológicas y la imagenología son necesarios y de gran utilidad para el diagnóstico, especialmente en niños con alto riesgo de progresión, siendo estos pacientes inmunocomprometidos, con desnutrición, pobreza y hacinamiento.

\section{REFERENCIAS}

Agrons, G., Markowitz, R. y Kramer, S. (1993). Pulmonary Tuberculosis in Children. Seminars in Roentgenology. 2;(2):158-172. Estados Unidos. https://doi.org/10.1016/ S0037-198X(05)80105-1

Barrera, C., Morales, D. (2017). Enfermedad de Pott: a propósito de un caso Pott's disease. A case report. Biociencias. 12(2):143-148. Colombia. https://doi. org/10.18041/2390-0512/bioc. .2.2296

Benzagmout, M., Boujraf, S., Chakour, K., Chaoui, M. (2011). Pott's disease in children. Surg Neurol Int. 2(1):1. Estados Unidos. https: //doi.org/10.4103/21527806.75459

Carrol, E., Clark, J. y Cant, A. (2001). Non-pulmonary tuberculosis. Paediatric respiratory reviews. (2):113-119. Inglaterra. https://doi.org/10.1053/prrv.2000.0118

Chang C, Yu C, Chih L et al. (2016). Early diagnosis of spinal tuberculosis. Journal of the Formosan Medical Association. 115(10):825-836. China. https://doi.org/10.1016/j.jfma.2016.07.001

Cortez, N., Delgado, J., Galdos, O. y Huicho, L. (2018). Reporte de caso de enfermedad de Pott en vértebras torácicas altas en un niño de dos años. Rev Peru Med Exp Salud Pública. 35(1):150. Perú. https:// doi.org/10.17843/rpmesp.2018.351.3564

Departamento de Epidemiología/MSPAS. Boletín de la Semana Epidemiológica SEMEPI No.13. Guatemala: MSPAS; Report No.: No. 13. Guatemala.

Fanlo, P., Tiberio, G. (2007). Tuberculosis extrapulmonar. An Sist Sanit Navar 30;(2):143- 162. España. https://doi. org/10.4321/S1137-66272007000400011

González, N., Macías, M., Hernández, M., Gutiérrez, P., Gómez, V., Juárez, H. (2014) Pulmonary Tuberculous: Symptoms, diagnosis and treatment. 19-year experience in a third level pediatric hospital. BMC Infect Dis. 14(1):401. México. https: / /doi.org/10.1186/1471-2334-14401

Macías, M. (2017). Tuberculosis pediátrica. Bol Méd Hosp Infant México. 74(1):1-2. México. https:// doi.org/10.1016/j.bmhimx.2017.01.003

Mellado, M., Santiago, B., Baquero, F., Moreno, D., Piñeiro, R., et al. (2018) Actualización del tratamiento de la tuberculosis en niños. An Pediatría. 88(1):52. España. https://doi.org/10.1016/j.anpedi.2017.05.013

Méndez, A., Baquero, F. (2016). Tuberculosis Pulmonar. Pediatr Integral. 20(2):10918. España.

Ramírez, M., Menéndez, A., Noguerado, A. (2015) Tuberculosis extrapulmonar, una revisión. Rev Esp Sanid Penit. 17(1):3-11. España. https://doi.org/10.4321/S157506202015000100002 
SOBRE AUTORAS

Dra. Gabriela Corleto Molina

Es Médica y Cirujana del Centro Universitario del Norte -CUNOR, de la Universidad San Carlos de Guatemala.

Dra. Erza López España

Es Médica y Cirujana, del Centro Universitario del Norte -CUNOR, de la Universidad San Carlos de Guatemala.

\section{Mtra. Celeste González Morales}

Es Médica y Cirujana de la Facultad de Ciencias Médicas, USAC, Maestría en Pediatría por la Universidad de San Carlos de Guatemala y Maestría en docencia universitaria con Énfasis en Andragogía (Cierre de Pensum) en el Centro Universitario del Norte USAC. Profesor interino del curso de pediatría del pregrado de la Carrera de Médica y Cirujano del CUNOR.

Conflicto de intereses

Declara no tener ningún conflicto de intereses.

Declaración de consentimiento informado

El estudio se realizó, respetando el Código de ética y buenas prácticas editoriales de publicación.

Derechos de uso

Copyright@ 2021 por Gabriela Corleto Molina, Erza López España y Celeste González Morales

Este texto está protegido por una licencia Creative Commons 4.0. Internacional

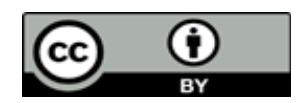

Usted es libre para compartir, copiar y redistribuir el material en cualquier medio o formato y adaptar el documento, remezclar, transformar y crear a partir del material para cualquier propósito, incluso comercialmente, siempre que cumpla la condición de atribución: usted debe reconocer el crédito de una obra de manera adecuada, proporcionar un enlace a la licencia, e indicar si se han realizado cambios. Puede hacerlo en cualquier forma razonable, pero no de forma tal que sugiera que tiene el apoyo del licenciante o lo recibe por el uso que hace. 